\title{
Complete genome characterization and evolutionary analysis of serotype -4 associated with severe dengue
}

\author{
K. VADDADI $\uparrow, C$. GANDIKOTA $\uparrow$ AND M. VENKATARAMANA* \\ Department of Biotechnology and Bioinformatics, School of Life Sciences, University of Hyderabad, Hyderabad, \\ Telangana State, India
}

Received 8 October 2016; Accepted 18 January 2017;

first published online 20 February 2017

\section{SUMMARY}

Dengue virus circulates as four independent serotypes posing a major public health threat around the globe. In the recent years, frequent dengue outbreaks are being reported in many parts of the world including India. Among four serotypes, Den-4 is the least sampled and studied serotype until recent times, but the reported cases with Den-4 infections were mostly known to associate with severe dengue. In the past three decades, only one complete genome sequence of Den-4 has been published from India. Hence there is a deficit in information with reference to this serotype which would be required in deciphering its association with severe dengue. In this study, we have carried out the complete genome characterization of Den-4 virus, isolated from a dengue shock syndrome patient during the 2015 outbreak from Hyderabad, South India. Phylogenetic analysis revealed the circulation of genotype I (lineage $C$ ) which showed close relatedness to the reported virulent strains. The data also indicated few unique amino acid substitutions which are known to be important in virus replication and epitope presentation. This is the first report of complete genome characterization of Den-4 from South India, which may assist in shaping the genetic diversity of circulating strains in India.

Key words: Dengue fever, dengue hemorrhagic fever (DHF), dengue serotype 4, dengue shock syndrome (DSS), Flaviviruses.

\section{INTRODUCTION}

Dengue is one of the leading arthropod-borne viral infections in the world transmitted by the bite of Aedes mosquitoes. This disease ranges from mild dengue fever to complicate dengue hemorrhagic fever (DHF) and dengue shock syndrome (DSS) [1]. Tropical and sub-tropical regions are the most affected parts of the globe. About 390 million people

\footnotetext{
* Author for correspondence: Assistant Professor M. Venkataramana, Department of Biotechnology and Bioinformatics, School of Life Sciences, University of Hyderabad, Hyderabad 500046, Telangana State, India.

(Email: mvrsl@uohyd.ernet.in)

$\dagger$ These authors contributed equally to this paper.
}

are being infected with dengue annually with nearly 500000 severe dengue cases [2]. Dengue incidences have been showing a steep increase in the recent years and the year 2015 was distinguished with the largest dengue outbreaks worldwide. India is one among the Southeast Asian countries which holds a big share of dengue viral infections. Delhi, the capital city of India, faced the worst dengue outbreak in 2015 with more than 15000 cases [3].

Dengue virus is a single-stranded positive sense RNA virus with $11 \mathrm{~kb}$ genome size that belongs to the genus Flavivirus of the family Flaviviridae. The genome constitutes three structural (capsid, membrane, and envelope) and seven non-structural (NS1, NS2A, NS2B, NS3, NS4A, NS4B, and NS5) genes 
flanked by untranslated regions (UTR) on both ends [4]. Dengue is caused by four immunologically related serotypes (Den-1, 2, 3, and 4). All four dengue virus serotypes were further resolved into different genotypes $[5,6]$. A change in circulating genotypes has been reported in the recent years from many countries, which may be due to global travel and virus transmission [7]. A shift in circulating genotypes or even lineages in a geographical region may increase the incidence of severe dengue cases [8]. Den-4 is the most diverged serotype with less prevalence. In spite of its less frequency, Den-4 was responsible for more DHF cases in children and also known to associate mostly with co-infections [9].

Although India is considered as hyperendemic of dengue infections, the dengue virus outbreaks were mostly dominated by serotypes 1 and 2 [10-12]. After the absence for three decades, Den-4 was reported from Delhi in 2003, Hyderabad in 2007, Pune in 2009 and Kerala in 2010 [13-15]. Epidemiological studies carried out during four years span from 2011 to 2014 in Delhi, reported the occurrence of all dengue serotypes except Den-4 [16]. The earlier studies regarding Den-4 in the country were solely based on the selected gene segments [13-17]. The complete Den-4 sequences available from India are of 1961 and 1962 strains, which belong to genotype $\mathrm{V}$. But the recently characterized strain isolated in 2009 belongs to the lineage $\mathrm{C}$ of genotype I [18]. After that, there are no reports on complete characterization of Den-4 from India. Our studies on the characterization of dengue serotypes occurred in Hyderabad during 2014-2015 revealed the circulation of all four serotypes with many co-infections and Den-4 serotype was found to be circulating evenly along with other serotypes. In 2014, out of 33 serotyped samples, Den-4 was identified in total 11 cases with four single infections and seven co-infections. Out of 11 , nine cases $(81 \cdot 6 \%)$ were of severe dengue (DHF/DSS) which explains the severity of disease caused by Den-4 (unpublished data). Hence to elaborate the knowledge of circulating Den-4 serotypes/ genotypes and their genetic diversity in India, the present study aimed at the complete genome characterization of Den-4, which is for the first time from South India.

\section{METHODS}

\section{Ethics statement}

Approval for the present study was obtained from the Institutional Ethics Committee, University of Hyderabad, Hyderabad, India (UH/IEC/2015/82).

\section{Sample collection}

Dengue virus-infected serum samples were collected from a tertiary care children's hospital located in Hyderabad, India, during 2014 and 2015. Total 160 samples were tested positive for dengue by NS1 antigen detection test and IgM/IgG capture ELISA. The dengue-positive samples were serotyped and one sample with serotype-4 infection was chosen for further analysis. The sample employed in this study belongs to an infant aged 8 months suffering from DSS. The sample and clinical information of the patient were obtained from the hospital with informed consent from the patient's parents.

\section{RNA extraction and RT-PCR}

Viral RNA was extracted from the serum sample using QIAamp viral RNA mini kit as per the manufacturer's instructions. For serotype detection, conventional seminested PCR was performed [19]. For whole genome sequencing, Superscript III first-strand synthesis kit was employed to synthesize the cDNAs using genespecific reverse primers. Eighteen primer sets were used to amplify the overlapping amplicons (Supplementary Table S1). Q5 high-fidelity DNA polymerase (New England Biolabs) was used for amplifications. Thermocycling conditions followed for gene amplifications were, initial denaturation at $98^{\circ} \mathrm{C}$ for $30 \mathrm{~s}$, followed by 35 cycles of denaturation at $98^{\circ} \mathrm{C}$ for $10 \mathrm{~s}$, annealing at $62-68{ }^{\circ} \mathrm{C}$ for $30 \mathrm{~s}$, extension at $72{ }^{\circ} \mathrm{C}$ for $30 \mathrm{~s}$ and a final extension at $72{ }^{\circ} \mathrm{C}$ for $2 \mathrm{~min}$.

\section{Cloning and sequencing}

The amplified products were gel purified using Qiagen gel extraction kit and cloned into CloneJET PCR cloning vectors according to the manufacturer's instructions. The cloned inserts were sequenced three times by a commercial sequencing company (Sandor Proteomics Pvt. Ltd). The obtained sequences were assembled using BioEdit 6.0.7 software and were submitted to the GenBank database.

\section{Phylogenetic and mutational analyses}

The complete open reading frame (ORF) region was used for phylogenetic analysis by employing the software MEGA $6 \cdot 0$ [20]. In this method, the complete sequence of the present strain was aligned and compared with the published Den-4 sequences in GenBank using Clustal W. Total 24 sequences across 
all genotypes of Den-4 were employed in construction of the phylogenetic tree. Then, the phylogenetic tree was constructed using the maximum-likelihood method based on the GTR (general time reversible) model with $\gamma$ distribution and invariant sites with 1000 bootstrap replications. BioEdit software was used to identify the unique mutations at nucleotide and amino acid level.

\section{T-cell epitope prediction}

T-cell epitopes were predicted for the capsid, envelope, NS3 and NS5 genes of prototype Philippines 1956 strain using the EpiJen online server [21].These epitopes were predicted for 18 different HLA alleles using appropriate TAP cut-off and proteasomal values. The predicted epitopes were mapped onto the identified unique mutations of the present strain.

\section{Selection pressure analyses}

Genome-wide positively selected sites were identified by using the online adaptive evolution server http://www. datamonkey.org/ [22]. The ratio of non-synonymous to synonymous substitutions (dN/dS) per codon sites were estimated using four different likelihood methods viz., SLAC (single likelihood ancestor counting), fixed effects likelihood (FEL), mixed effects model of evolution (MEME) and fast unbiased Bayesian approximate (FUBAR). The analysis was run based on neighborjoining tree and significant $P$-value $(<0 \cdot 1)$.

\section{RESULTS}

\section{Clinical presentation}

In the present study, dengue infected sample was collected from an 8-month-old male infant who does not have any previous history of dengue. The sample was tested positive for dengue virus by NS1 antigen detection test, and Den-4 was found to be the infecting serotype. The patient was presented with severe disease symptoms pertaining to DSS as per the WHO criteria [23] and required ICU level of treatment for 15 days. At the time of admission, he was febrile $\left(101 \cdot 3^{\circ} \mathrm{F}\right)$ with symptoms of maculopapular rash all over the body, cold, cough, vomiting, and diminished air entry on the right side. He developed complications such as worsening thrombocytopenia, leukocytosis, anemia, deranged coagulation profile, endotracheal bleed, mild ascites with gallbladder edema, pleural effusion, multi-organ dysfunction syndrome, and ventilator-associated pneumonia. Platelet counts of the patient from the first day of admission were ranged as follows: 202000,69 000, 24 000, $97000,72000,106000,112000,84000$, 66000,104 000, and 207000 . Platelet transfusion was given on the third day. Later he developed the signs of shock in the form of prolonged CFT (capillary filling time), flushed peripheries, tachycardia, and worsening respiratory distress.

\section{Sequence analysis}

The viral RNA extracted from the patient serum sample was used for serotyping and complete genome amplification. Serotyping analysis revealed the presence of Den-4 serotype in the sample. For whole genome sequencing, 18 overlapping amplifications were carried out in order to get the complete virus genome (Supplementary Fig. S1). The complete genome sequence of the present isolate $\mathrm{UOH}_{-} 23916$ constitutes $10652 \mathrm{nts}$ with 5'UTR of $96 \mathrm{nts}, 3^{\prime} \mathrm{UTR}$ of $388 \mathrm{nts}$ and with an ORF of $10162 \mathrm{nts}$ coding for 3387 amino acids. The sequence details were submitted to GenBank under the accession number 'KX845005'. The nucleotide sequence comparison of the present strain revealed 99\% identity with the Indian 2009 strain (isolate from Pune), 98\% with Pakistan 2009 strain and 95-96\% with the strains of lineage B of the same genotype. $5^{\prime}$ UTR was found to be highly conserved across the genotype, whereas a few deletions and substitutions were identified in $3^{\prime} \mathrm{UTR}$. Thirteen nucleotide deletion was observed in the variable region of all the recent strains including the present strain when compared with the prototype strains of 1956 and 1961. Two unique nucleotide substitutions T163G and C211A were observed in the 3'UTR of the present strain (Supplementary Fig. S2).

\section{Phylogenetic analysis}

The phylogenetic tree of Den-4 serotype was resolved into five genotypes, with the present strain grouped into genotype I (Southeast Asia). Genotype I constitute three lineages $\mathrm{A}, \mathrm{B}$, and $\mathrm{C}$, among which the lineage $C$ represents the recent Indian isolates and Pakistan 2009 strain [24]. The old Indian isolates of 1961 and 1962 were genetically distinct and clustered into genotype V. Genotype II (Southeast Asia and Americas) constitutes strains from Venezuela, Puerto Rico, Thailand, and Taiwan and only two Thailand strains grouped into genotype III (Thailand). Genotype IV (sylvatic) constitutes the strains isolated from primates in Malaysia (Fig. 1). 


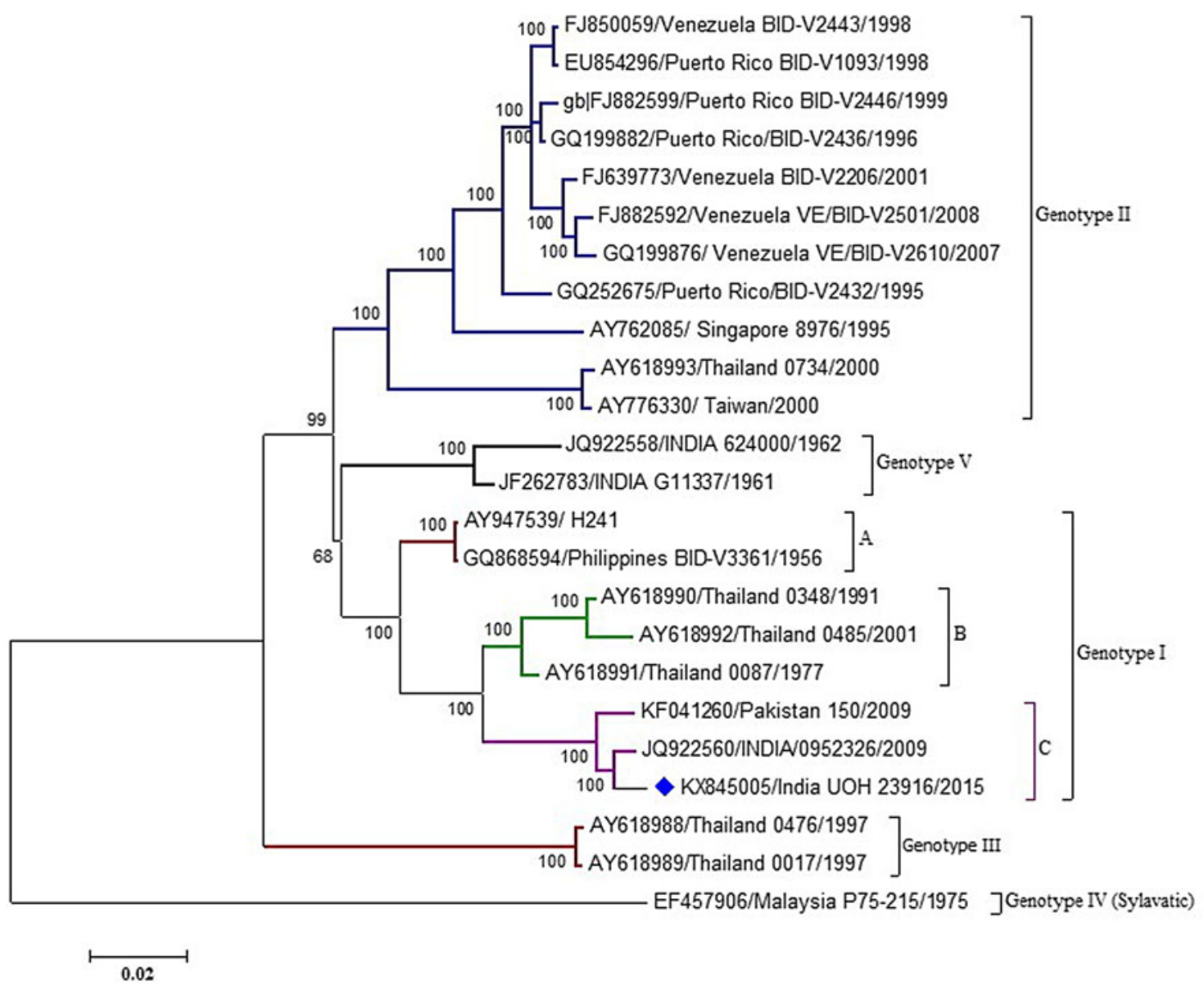

Fig. 1. Phylogenetic tree of Den-4 strains generated by maximum-likelihood method based on the complete ORF sequence (10 $162 \mathrm{nts})$. Bootstrap values are indicated. The scale bar represents the number of nucleotide substitutions per site. GenBank accession number, name, place, and year of isolation are also indicated. The present strain in the study is indicated with colored block.

\section{Amino acid sequence analysis}

The amino acid sequences of the present strain were aligned with existing Indian strains and closely related foreign strains to identify the substitutions across the coding region. Six amino acid substitutions (M102T of capsid; V208I of NS3; V18A of NS4B; H201Y, $\mathbf{K} 638 \mathbf{E}$, and $\mathbf{Q} 870 \mathbf{H}$ of NS5) were identified to be unique to the present strain. A total of 19 amino acid changes were found to be specific to the Lineage $\mathrm{C}$ of Genotype I which constitutes strains of India 2009 and Pakistan 2009 along with the present sequenced 2015 strain. Apart from the above substitutions, the present strain shares three similar substitutions with the older 1956 and 1961 strains (T221A, L429F of envelope and R631Q of NS5) (Table 1). The observed unique amino acids in the present strain were mapped with the T-cell epitopes and found that these amino acids were involved in T-cell epitope presentation for a maximum number of HLA alleles as shown in Table 2 .

\section{Selection pressure analysis}

To analyze the evolutionary force undergone by the virus, we performed selection pressure analysis across the coding region of the genome. A dataset of six strains from three lineages of genotype I along with one Indian prototype strain (India-G11337 1961) was used for the analysis. Most of the codon sites of the present strain were found to be under strong purifying selection which is similar to the earlier reports [25]. Fifteen sites were identified as positively selected by two methods (FEL and MEME), out of which one site was identified in NS5 (R3118Q) by both methods and 14 were identified by MEME method. Among these, four sites from structural proteins and 10 sites 
Table 1. Unique amino acid substitutions observed in the Hyderabad strain ( $U o H)$ and the corresponding residues in the reference strain Philippines/BID-V3361/1956, and the closely related Den-4 strains

\begin{tabular}{|c|c|c|c|c|c|c|c|c|c|}
\hline S. no. & $\begin{array}{l}\text { AA } \\
\text { position } \\
\text { (ORF) }\end{array}$ & $\begin{array}{l}\text { AA } \\
\text { position } \\
\text { (Protein) }\end{array}$ & $\begin{array}{l}\text { BID-V3361 } \\
\text { Philippines } \\
\text { (1956) }\end{array}$ & $\begin{array}{l}\text { G11337 } \\
\text { India } \\
\text { (1961) }\end{array}$ & $\begin{array}{l}0087 \\
\text { Thailand } \\
\text { (1977) }\end{array}$ & $\begin{array}{l}0485 \\
\text { Thailand } \\
\text { (2001) }\end{array}$ & $\begin{array}{l}1150 \\
\text { Pakistan } \\
(2009)\end{array}$ & $\begin{array}{l}\text { Pune } \\
0952326 \\
\text { India } \\
(2009)\end{array}$ & $\begin{array}{l}\text { UoH } \\
23916 \\
\text { India } \\
\text { (2015) }\end{array}$ \\
\hline \multicolumn{10}{|c|}{ Capsid } \\
\hline $\begin{array}{l}1 \\
\text { prM }\end{array}$ & 102 & 102 & $\mathbf{M}$ & $\mathbf{V}$ & I & I & I & I & $\mathbf{T}$ \\
\hline 2 & 135 & 22 & $\mathrm{P}$ & & & & & $\mathrm{R}$ & \\
\hline 3 & 165 & 52 & E & K & K & $\mathrm{K}$ & $\mathrm{K}$ & $\mathrm{K}$ & $\mathrm{K}$ \\
\hline \multicolumn{10}{|l|}{ M } \\
\hline 4 & 269 & 66 & V & V & I & I & I & I & I \\
\hline \multicolumn{10}{|l|}{ ENV } \\
\hline 5 & 409 & 130 & $V$ & . & . & . & $I$ & $I$ & $I$ \\
\hline 6 & 443 & 164 & I & . & . & . & $\mathrm{T}$ & . & . \\
\hline 7 & 453 & 174 & $K$ & . & . & . & $E$ & $E$ & $E$ \\
\hline 8 & 481 & 202 & $K$ & . & . & . & $N$ & $N$ & $N$ \\
\hline 9 & 500 & 221 & A & $\mathrm{T}$ & $\mathrm{T}$ & $\mathrm{T}$ & $\mathrm{T}$ & . & . \\
\hline 10 & 506 & 227 & $\mathrm{~S}$ & . & $\mathrm{L}$ & $\mathrm{L}$ & . & . & . \\
\hline 11 & 550 & 271 & D & . & . & . & . & $\mathrm{L}$ & . \\
\hline 12 & 556 & 277 & $\mathrm{H}$ & . & . & . & . & $\mathrm{L}$ & . \\
\hline 13 & 630 & 351 & $I$ & & 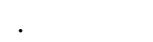 & & & V & $V$ \\
\hline 14 & 639 & 360 & Y & $\mathrm{N}$ & $\mathrm{N}$ & $\mathrm{N}$ & $\mathrm{N}$ & $\mathrm{N}$ & $\mathrm{N}$ \\
\hline 15 & 672 & 393 & $\mathrm{R}$ & & . & . & & $\mathrm{T}$ & \\
\hline 16 & 708 & 429 & $\mathbf{L}$ & F & . & & & . & $\mathbf{F}$ \\
\hline 17 & 740 & 461 & $\mathrm{~F}$ & $\mathrm{~L}$ & . & $\mathrm{L}$ & & . & . \\
\hline \multicolumn{10}{|l|}{ NS1 } \\
\hline 18 & 795 & 21 & V & . & . & 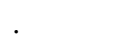 & $I$ & $I$ & $I$ \\
\hline 19 & 902 & 128 & A & A & $\mathrm{T}$ & $\mathrm{T}$ & $\mathrm{T}$ & $\mathrm{T}$ & $\mathrm{T}$ \\
\hline 20 & 952 & 178 & V & . & . & . & . & $\mathrm{M}$ & . \\
\hline \multicolumn{10}{|l|}{ NS2A } \\
\hline 21 & 1188 & 59 & $D$ & . & . & . & $N$ & $N$ & $N$ \\
\hline 22 & 1192 & 63 & $G$ & . & $S$ & . & $S$ & $S$ & $S$ \\
\hline 23 & 1198 & 69 & I & . & . & . & . & $\mathrm{T}$ & . \\
\hline 24 & 1249 & 120 & I & . & . & . & . & V & V \\
\hline \multirow{2}{*}{\multicolumn{10}{|c|}{ NS2B }} \\
\hline & & & & & & & & & \\
\hline 26 & 1402 & 55 & $N$ & . & . & . & $T$ & $T$ & $T$ \\
\hline \multicolumn{10}{|l|}{ NS3 } \\
\hline 27 & 1487 & 13 & A & . & . & & $\mathrm{T}$ & . & . \\
\hline 28 & 1516 & 42 & M & . & . & $\mathrm{T}$ & & & \\
\hline 29 & 1536 & 62 & $\mathrm{~T}$ & . & $\mathrm{S}$ & S & $\mathrm{S}$ & $\mathrm{S}$ & S \\
\hline 30 & 1574 & 100 & $I$ & 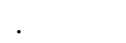 & . & & $V$ & $V$ & $V$ \\
\hline 31 & 1645 & 171 & $\mathrm{~T}$ & I & I & I & I & I & I \\
\hline 32 & 1682 & 208 & V & . & . & & & & I \\
\hline 33 & 1795 & 321 & A & . & $\mathrm{T}$ & $\mathrm{T}$ & $\mathrm{T}$ & $\mathrm{T}$ & $\mathrm{T}$ \\
\hline 34 & 1857 & 383 & $I$ & . & . & . & $V$ & $V$ & $V$ \\
\hline 35 & 1908 & 434 & $S$ & $L$ & $L$ & $L$ & $I$ & $I$ & $I$ \\
\hline 36 & 2079 & 605 & $M$ & . & . & . & $V$ & $V$ & $V$ \\
\hline \multicolumn{10}{|c|}{ NS4A } \\
\hline 37 & 2170 & 78 & $I$ & . & . & . & $M$ & $M$ & $M$ \\
\hline 38 & 2209 & 117 & $V$ & $A$ & . & . & $A$ & $A$ & $A$ \\
\hline 39 & 2240 & 148 & I & . & $\mathrm{V}$ & $\mathrm{V}$ & $\mathrm{V}$ & V & V \\
\hline \multicolumn{10}{|c|}{ NS4B } \\
\hline 40 & 2260 & 18 & V & . & . & . & & $\mathbf{E}$ & $\mathbf{A}$ \\
\hline 41 & 2482 & 240 & $A$ & . & . & & $T$ & $T$ & $T$ \\
\hline
\end{tabular}


Table 1 (cont.)

\begin{tabular}{|c|c|c|c|c|c|c|c|c|c|}
\hline S. no. & $\begin{array}{l}\text { AA } \\
\text { position } \\
\text { (ORF) }\end{array}$ & $\begin{array}{l}\text { AA } \\
\text { position } \\
\text { (Protein) }\end{array}$ & $\begin{array}{l}\text { BID-V3361 } \\
\text { Philippines } \\
\text { (1956) }\end{array}$ & $\begin{array}{l}\text { G11337 } \\
\text { India } \\
(1961)\end{array}$ & $\begin{array}{l}0087 \\
\text { Thailand } \\
\text { (1977) }\end{array}$ & $\begin{array}{l}0485 \\
\text { Thailand } \\
\text { (2001) }\end{array}$ & $\begin{array}{l}1150 \\
\text { Pakistan } \\
(2009)\end{array}$ & $\begin{array}{l}\text { Pune } \\
0952326 \\
\text { India } \\
(2009)\end{array}$ & $\begin{array}{l}\text { UoH } \\
23916 \\
\text { India } \\
(2015)\end{array}$ \\
\hline \multicolumn{10}{|l|}{ NS5 } \\
\hline 42 & 2688 & 201 & $\mathbf{H}$ & . & . & . & . & & $\mathbf{Y}$ \\
\hline 43 & 2713 & 226 & $G$ & . & . & . & . & $R$ & $R$ \\
\hline 44 & 2434 & 247 & $H$ & . & . & . & $Y$ & $Y$ & $Y$ \\
\hline 45 & 2762 & 275 & $T$ & . & . & . & $A$ & $A$ & $A$ \\
\hline 46 & 2860 & 373 & $V$ & . & . & . & $I$ & $I$ & $I$ \\
\hline 47 & 3118 & 631 & $Q$ & 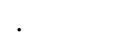 & $\mathrm{R}$ & $\mathrm{R}$ & $\mathrm{R}$ & $\mathrm{R}$ & . \\
\hline 48 & 3125 & 638 & $\mathbf{K}$ & & . & . & & 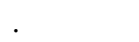 & $\mathbf{E}$ \\
\hline 49 & 3357 & 870 & $\mathbf{Q}$ & & . & . & & & $\mathbf{H}$ \\
\hline 50 & 3364 & 877 & $\mathrm{~K}$ & & . & . & & I & . \\
\hline
\end{tabular}

Unique amino acid substitutions identified in $\mathrm{UoH}$ strains are denoted by bold letters and substitutions specific to the lineage $\mathrm{C}$ strains are indicated in italics.

Table 2. Mapping of genome-wide unique mutations with the predicted T-cell epitopes

\begin{tabular}{lcll}
\hline \hline Mutation & Position & Epitope & HLA alleles \\
\hline Capsid & & & \\
M102T & 98 & KRSTMTLLC & HLA-A*0101, HLA-A*07 \\
M102T & 100 & STMTLLCLI & HLA-A*0101, HLA-A*0201, HLA-A*6802, HLA-A*53 \\
M102T & 102 & MTLLCLIPT & HLA-A*0101, HLA-A*6802 \\
Envelope & & & \\
L429F & 424 & SVGGLLTSL & HLA-A*07, HLA-A*3501, HLA-A*6802 \\
L429F & 426 & GGLLTSLGK & HLA-A*0301, HLA-A*1101, HLA-A*6801 \\
L429F & 427 & LLTSLGKAV & HLA-A*0201, HLA-A*0203, HLA-A*3101 \\
L429F & 428 & & HLA-A*0201, HLA-A*0206, HLA-A*0202, HLA-A*0203 \\
NS3 & & & \\
V208I & 204 & LPSIVREAL & HLA-A*07, HLA-A*3501, HLA-A*51, HLA-A*53 \\
V208I & 206 & SIVREALKR & HLA-A*0101, HLA-A*0301, HLA-A*03101 \\
V208I & 207 & IVREALKRR & HLA-A*3101, HLA-A*6801 \\
NS5 & & & \\
H201Y & 197 & LQRKHGGSL & HLA-A*0206 \\
K638E & 633 & DMHNPKGLK & HLA-A*0301, HLA-A*1101 \\
K638E & 636 & NPKGLKERV & HLA-A*07, HLA-A*51 \\
Q870H & 863 & NIHDAITQV & HLA-A*0202, HLA-A*0206 \\
Q870H & 869 & TQVRNLIGK & HLA-A*0301, HLA-A*1101 \\
\hline \hline
\end{tabular}

Position of the epitope is with reference to the proteins of dengue virus prototype strain (Philippines 1956).

from the non-structural proteins (NS3 and NS5) were found to be under positive selection.

\section{DISCUSSION}

Dengue infection has the long-standing history especially with reference to the severity and the strains involved. It is evident from the literature that dengue virus serotypes 1 and 2 were dominated the dengue epidemics in different countries for decades. In recent times, dengue is hyperendemic in many countries including India caused by all four serotypes [12, 26]. In Southeast Asian countries like India, severe dengue is most common in the children. Earlier studies from the countries like India, Sri Lanka, and Thailand reported severe dengue complications in the case of infants [26-30]. Incidences of Den-4 are known to be increased recently which are also associated with severe dengue and even primary infections with Den-4 were reported to cause DHF in children [25]. 
Apart from causing severe dengue, Den-4 was also known to be mostly involved in co-infections. In co-infections involved by Den-4 and 1 in mosquito species Den-4 seems to be availed competitive advantage for its multiplication [31]. Our earlier studies also indicated that all the Den- 4 co-infected patients suffered from severe dengue (unpublished data).The patient in the present study is an infant of 8 months old with DSS with the primary infection.

Research groups have predicted that Den-4 viruses might have originated from India and then probably exported to different countries. Den-4 viruses were first identified in India during the 1960s which were more genetically distinct from other genotypes and were placed under genotype V. However, these strains were extinct at present. The characterized strain in the present study clustered within the lineage $\mathrm{C}$ of genotype I along with India 2009 and Pakistan 2009 strains (Fig. 1). Genotype I was referred as an evolving lineage because of the highest genomic diversity within the genotype and more virulent strains with high dissemination potential [7].

As dengue is an RNA virus it accumulates many mutations across the genome, but relatively less when compared with other RNA viruses. Fifteen positively selected sites were identified in the present strain which are mostly restricted to the non-structural genes. Reports indicate the strong purifying selection and low evolutionary rate in dengue viruses [32]. In the present study, positive selection was observed mainly in NS3 and NS5 genes along with envelope gene. Apart from the positively selected sites, a few unique substitutions were also identified in which one non-conservative mutation M102T was located in capsid protein. Earlier reports indicate that 102107 amino acids of the capsid act as a degradation motif to nullify the antiviral activity of $h S e c 3 p$ by inducing its degradation and allowing efficient binding of elongation factor- $1 \alpha$ with viral genome for virus particle production [33]. Two conservative mutations were identified one each in NS3 and NS4B and three non-conservative mutations in NS5. These unique mutations were correlated with the predicted T-cell epitopes which were mostly found to be involved in T-cell epitope presentation. India 2009 strain was found to be closely related to the present strain with reference to the amino acid diversity and also reported to be involved in severe dengue. These two strains share three common amino acid substitutions at envelope (I351V), NS2A (I120V), and NS5 (G226R) proteins. In addition, 19 amino acid substitutions were found to be common in India 2009, Pakistan 2009 and in the present strain (Table 1). This observation indicates the fact that these mutations may benefit the virus in escaping the host adaptive immunity as supported by earlier literature [32, 34]. The geographical area where the present strain is isolated is known for the circulation of Den-4 of which the pathogenicity is not clear [13]. It has been postulated that high virulent strains exploit increased ADE (antibodydependent enhancement) in the geographical areas in which the low virulent strains of the same serotype/ genotype circulated. Hence, we presume that the strain in the present study may have used the similar mechanism and caused the severe dengue. The variable region of 3'UTR tends to undergo many changes including substitutions or deletions, such as a 13 nucleotide deletion observed in all the recent Den-4 strains along with the present strain. However, there is no clarity regarding the role of these mutations in disease severity or virulence and hence remains to be further elucidated.

In summary, this is the first study regarding the complete genome characterization of Den-4 from South India. We identified the circulation of genotype I strain that formed a unique lineage along with recent Indian and Pakistan strains of 2009. A few unique substitutions that constitute the $\mathrm{T}$-cell epitopes were identified in the present strain. A positive selection pressure was also found to be acting on few amino acid sites. Our findings along with the earlier reports suggest that Den-4 genotype I is an evolving genotype which needs a continuous monitoring to understand the dynamics of its circulation, evolution, and severity of the disease in future outbreaks and hence to formulate the proper control measures.

\section{SUPPLEMENTARY MATERIAL}

The supplementary material for this article can be found at https://doi.org/10.1017/S0950268817000243.

\section{ACKNOWLEDGEMENTS}

The authors are grateful to the University Grant Commission (UGC) (Grant No. 41-518/2012(SR)), UPE I\&II, University of Hyderabad (UH/LS/UPE2/28/2015) and Department of Biotechnology (DBT, India), (BT/PR14240/MED/29/985/2015) for the financial support. K.V. gets a fellowship from UGC and C. G. gets from DBT, India. The authors are also thankful 
to the CEO of Lotus children's hospital, Hyderabad for providing clinical samples.

\section{DECLARATION OF INTEREST}

None.

\section{REFERENCES}

1. Holmes EC, Burch SS. The causes and consequences of genetic variation in dengue virus. Trends in Microbiology 2000; 8: 74-77.

2. Bhatt S, et al. The global distribution and burden of dengue. Nature 2013; 496: 504-507.

3. WHO. Dengue and severe dengue. Fact sheet no. 117, July 2016 (http://www.who.int/mediacentre/factsheets/ fs117/en/index.html). Accessed August 2016.

4. Leopoldo GG, Claudia VF, Andrea VG. Functional RNA elements in the dengue virus genome. Viruses 2011; 3: 1739-1756.

5. Holmes EC, Twiddy SS. The origin, emergence and evolutionary genetics of dengue virus. Infection Genetics and Evolution 2003; 3: 19-28.

6. Rico-Hesse R. Microevolution and virulence of dengue viruses. Advances in Virus Research 2003; 59: 315-341.

7. Huang JH, et al. Molecular characterization and phylogenetic analysis of dengue viruses imported into Taiwan during 2008-2010. American Journal of Tropical Medicine and Hygiene 2012; 87: 349-358.

8. Shrivastava A, et al. Lineage shift of dengue virus in Eastern India: an increased implication for DHF/DSS. Epidemiology and Infection 2015; 143: 1599-1605.

9. Nisalak A, et al. Serotype-specific dengue virus circulation and dengue disease in Bangkok, Thailand from 1973-1999. American Journal of Tropical Medicine and Hygiene 2003; 68: 191-202.

10. Dar L, et al. Co-circulation of dengue serotypes, Delhi, India, 2003. Emerging Infectious Diseases 2006; 12: 352-353.

11. Bharaj P, et al. Concurrent infections by all four dengue virus serotypes during an outbreak of dengue in 2006 in Delhi, India. Virology Journal 2008; 5: 1.

12. Nivedita G, et al. Dengue in India. Indian Journal of Medical Research 2012; 136: 373-390.

13. Dash PK, et al. Emergence of dengue virus type 4 (genotype I) in India. Epidemiology and Infection 2011; 139: 857-861.

14. Cecilia D, et al. Detection of dengue-4 virus in Pune, western India after an absence of 30 years - its association with two severe cases. Virology Journal 2011; 8: 46.

15. Kumar NP, et al. Genetic characterization of dengue viruses prevalent in Kerala State, India. Journal of Medical Microbiology 2013; 62: 545-552.

16. Afreen $\mathbf{N}$, et al. Phylogenetic and molecular clock analysis of dengue serotype 1 and 3 from New Delhi, India. PLoS ONE 2015; 10: e0141628.
17. Patil JA, et al. Influence of evolutionary events on the Indian subcontinent on the phylogeography of dengue type 3 and 4 viruses. Infection Genetics and Evolution 2012; 12: 1759-1769.

18. Rossi SL, et al. Genetic and phenotypic characterization of sylvatic dengue virus type 4 strains. Virology 2012; 423: 58-67.

19. Lanciotti RS, et al. Rapid detection and typing of dengue viruses from clinical samples by using reverse transcriptase-polymerase chain reaction. Journal of Clinical Microbiology 1992; 30: 545-551.

20. Tamura K, et al. MEGA 6: molecular evolutionary genetics analysis version 6.0. Molecular Biology and Evolution 2013; 30: 2725-2729.

21. Doytchinova IA, Guan P, Flower DR. EpiJen: a server for multistep $\mathrm{T}$ cell epitope prediction. $B M C$ Bioinformatics 2006; 7: 131.

22. Sergei LKP, Simon DWF. Datamonkey: rapid detection of selective pressure on individual sites of codon alignments. Bioinformatics 2005; 21: 2531-2533.

23. WHO. Dengue Guidelines for Diagnosis, Treatment, Prevention and Control. Geneva, 2009 (New edition). WHO/HTM/NTD/DEN/2009.1.

24. Koo C, et al. Evolution and heterogeneity of multiple serotypes of Dengue virus in Pakistan, 2006-2011. Virology Journal 2013; 10: 275.

25. Klungthong $\mathbf{C}$, et al. The molecular epidemiology of dengue virus serotype 4 in Bangkok, Thailand. Virology 2004; 329: 168-179.

26. Martins VdoC, et al. Clinical and virological descriptive study in the 2011 outbreak of dengue in the Amazonas, Brazil. PLOS ONE 2014; 9: e100535.

27. Aggarwal A, et al. An epidemic of dengue hemorrhagic fever and dengue shock syndrome in children in Delhi. Indian Pediatrics 1998; 35: 727-732.

28. Kabilan L, et al. Dengue disease spectrum among infants in the 2001 dengue epidemic in Chennai, Tamil Nadu, India. Journal of Clinical Microbiology 2003; 41: 3919-3921.

29. Pancharoen C, Thisyakorn U. Dengue virus infection during infancy. Translations of the Royal Society of Tropical Medicine and Hygiene 2001; 95: 307-308.

30. Jain A, Chaturvedi UC. Dengue in infants: an overview. FEMS Immunology and Medical Microbiology 2010; 59: 119-130.

31. Vazeille M, et al. Competitive advantage of a dengue 4 virus when co-infecting the mosquito Aedes aegypti with a dengue 1 virus. BMC Infectious Diseases 2016; 16: 318.

32. Chen R, Vasilakis N. Dengue - Quo tuetquovadis? Viruses 2011; 3: 1562-1608.

33. Bhuvanakantham R, Ng ML. West Nile virus and dengue virus capsid protein negates the antiviral activity of human Sec3 protein through the proteasome pathway. Cellular Microbiology 2013; 15: 1688-1706.

34. Afreen $\mathbf{N}$, et al. Evolutionary analysis of dengue serotype 2 viruses using phylogenetic and Bayesian methods from New Delhi, India. PLoS Neglected Tropical Diseases 2016; 10: e0004511. 